\title{
E15 HYSTERESIS IN THREE-PHASE POROUS MEDIA FLOW
}

\author{
B. PLOHR ${ }^{1}$, D. MARCHESIN ${ }^{2}$, P. BEDRIKOVETSKY ${ }^{3}$, J. E. ALTOÉ F ${ }^{3}$ AND A. J. DE SOUZA \\ ${ }^{1}$ Department of Mathematics, State University of New York at Stony Brook, Stony Brook, NY 11794-3651, USA \\ ${ }^{2}$ IMPA, Estrada Dona Castorina 110, 22460-320 Rio de Janeiro, RJ, Brazil \\ ${ }^{3}$ LENEP / UENF, Rod. Amaral Peixoto km 163 - Av. Brenand s/no., 27925-310 Macaé, RJ, Brazil \\ ${ }^{4}$ Universidade Federal de Campina Grande, Av. Aprigio Veloso 882, 58109-970 Campina Grande, PB, Brazil
}

\begin{abstract}
We consider a model for immiscible three-phase (e.g., water, oil, and gas) flow in a porous medium. We allow the relative permeability of the gas phase to exhibit hysteresis, in that it varies irreversibly along two extreme paths (the imbibition and drainage curves) that bound a region foliated by reversible paths (scanning curves). By numerically solving one-dimensional flow problems involving simultaneous and alternating injection of water and gas into a rock core, we demonstrate the effects of hysteresis.
\end{abstract}

\section{Introduction}

In this work, we consider the injection problem for a model of three-phase (water-oil-gas) flow in a core sample of porous rock, taking into account hysteresis effects in the relative permeability of the gas phase. Hysteresis may be the most important phenomenon in multiphase porous media flow that is lacking rigorous mathematical analysis.

Mathematically, hysteresis effects are modeled by assuming that (a) there are two extreme permeability curves for the gas, the imbibition and drainage curves, that describe the flow when the gas saturation is decreasing and increasing, respectively, in an irreversible fashion; and (b) there is a continuous family of permeability curves, called scanning curves, that lead between the imbibition and drainage curves and describe the flow when the gas permeability changes reversibly. These features are motivated by the experimental work of Braun-Holland [4].

For two-phase flow, the first mathematical model accounting for hysteresis effects was formulated in Ref. [9] and studied further in Ref. [10]. In these papers, only the imbibition and drainage permeability curves were modeled. A more complete model for two-phase flow, was proposed in Refs. [5,6,7] and studied further in Refs. [3,12]. In the present paper, we extend the latter model to three-phase flow and use the extension to begin to explore the effects of hysteresis on the Water-Alternating-Gas (WAG) oil-recovery process.

In outline, the remainder of the paper is as follows. In the next section, we recount the governing equations for three-phase flow. In Section 3, we describe the phenomenology of relative permeability hysteresis and explain how it can be modeled by introducing a hysteresis parameter. . In Section 4 we show that the asymptotic Riemann solution for this model in two-phase flow has the uncommon property of depending on small perturbations on the initial data. This model, which assumes that equilibrium is always maintained, is extended in Section 5 by allowing nonequilibrium states that relax quickly to equilibrium. In Section 6, we present the results of numerical experiments for one-dimensional three-phase flow, both with and without

\footnotetext{
$8^{\text {th }}$ European Conference on the Mathematics of Oil Recovery - Freiberg, Germany, 3 - 6 September 2002
} 
permeability hysteresis, for flow problems involving the injection of water and gas either simultaneously or alternately into a rock core. Finally, in Section 7, we summarize our results.

\section{The Governing Equations for Three-Phase Flow}

The equations governing flow of water, oil, and gas in a one-dimensional rock core, neglecting the effects of gravity, mass transfer, temperature, compressibility, and heterogeneity, take the nondimensional form

$$
\frac{\partial s_{\alpha}}{\partial t}+\frac{\partial f_{\alpha}}{\partial x}=D_{\alpha} \quad \text { for } \alpha=w, o, g
$$

Here: $x$ is position along the core and $t$ is time; $s_{\alpha}$ is the saturation of phase $\alpha ; f_{\alpha}$ is the fractional flow, which is given in terms of the relative permeability $k_{r \alpha}$ and the phase viscosity $\mu_{\alpha}$ by $f_{\alpha}=\lambda_{\alpha} / \lambda$, where $\lambda_{\alpha}=k_{r \alpha} / \mu_{\alpha}$ and $\lambda=\lambda_{w}+\lambda_{o}+\lambda_{g}$; and $D_{\alpha}$ is the diffusion term that arises because of capillary pressure differences. As $s_{w}+s_{o}+s_{g}=1$, only two of the three Eqs. (2.1) are independent, which generalize the Buckley-Leverett model for two-phase flow.

The relative permeabilities depend on the saturations. To be specific, we assume that the relative permeability functions for the wetting phase (water) and the intermediate wetting phase (oil) depend solely on their corresponding fluid saturations. On the other hand, we assume that the relative permeability of the non-wetting phase (gas) depends not only on the gas saturation but also on the direction in which this saturation is changing. This leads to hysteresis in the gas relative permeability, which we describe more fully in the next section. For the capillary pressure differences, we assume that $p_{w o}$, the water pressure minus the oil pressure, depends solely on $s_{w}$, and that $p_{g o}$, analogously defined, depends solely on $s_{g}$.

\section{The Scanning Hysteresis Model}

To visualize the hysteresis phenomenon in the relative permeability, consider Fig. 3.1(a), which plots $k_{r g}$ vs. $s_{w}+s_{o}=1-s_{g}$. (This plot is a caricature of the experiments of Braun-Holland [4].) During injection of oil or water, $k_{r g}$ falls gradually until a threshold is reached, at which stage $k_{r g}$ begins to decrease sharply. The latter stage is termed imbibition. If gas is subsequently injected, then $k_{r g}$ does not recover along the imbibition path; rather, it increases only gradually until another threshold is reached, whereupon it rises sharply. This second stage is called drainage, and the type of flow that occurs between the imbibition and drainage stages is called scanning flow. Changes in permeability during scanning flow are approximately reversible, whereas changes during drainage and imbibition are irreversible. Thus there is hysteresis, or memory, exhibited by the flow.

Our model for relative permeability hysteresis in three-phase flow is a straightforward extension of the two-phase model developed and studied in Refs. [5,6,7,3,12], which is called the Scanning Hysteresis Model (SHM). In this model, the empirical imbibition, drainage, and scanning curves, like those shown in Fig. 3.1(a), are idealized in the manner of Fig. 3.1(b). Also, to record the history of the flow, a hysteresis parameter $\pi$ is introduced. The value of $\pi$ at position $x$ at time $t$ can be viewed as representing the configuration of the three fluid phases within the pores, resulting from past evolution of the flow, in an infinitesimal volume surrounding that position at that time. During scanning flow, this configuration stays fixed, so that $\pi$ remains constant. Mathematically, therefore, $\pi$ simply labels the scanning curves. 

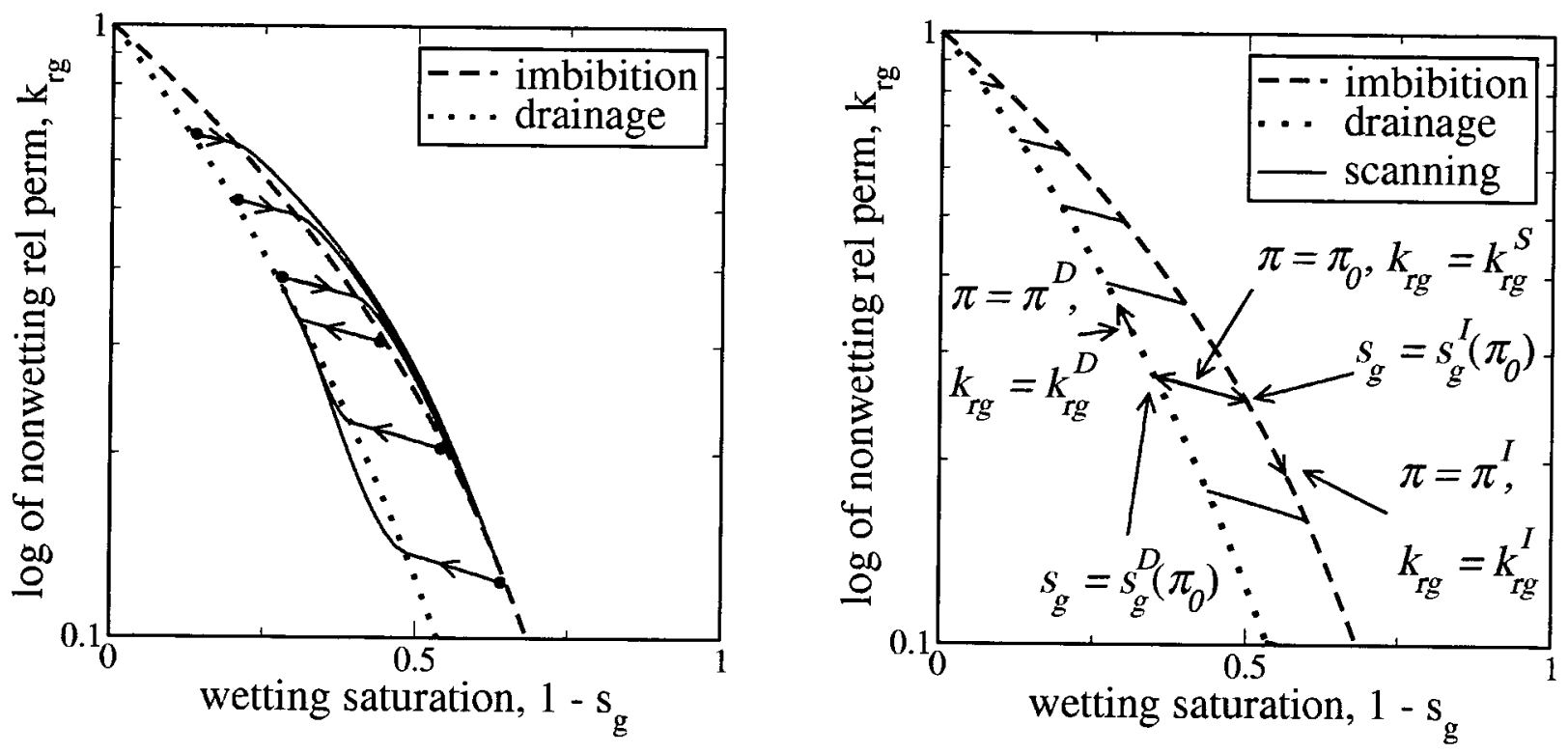

Figure 3.1. Relative permeability of the nonwetting phase as a function of the total saturation of the wetting phases. (a) During imbibition and drainage processes, the indicated directed paths are followed. (b) The imbibition, drainage and scanning curves, as idealized in the SHM.

Consider the case of scanning flow. Then, as seen in Fig. 3.1(b), $\pi$ has a certain constant value $\pi_{0}$, the gas saturation belongs to a limited range $s_{g}^{I}\left(\pi_{0}\right)<s_{g}<s_{g}^{D}\left(\pi_{0}\right)$, and the gas relative permeability is specified by the function $k_{r g}^{s}$ of $s_{g}$ that is determined by $\pi_{0}$. Thus

$$
k_{r g}=k_{r g}^{S}\left(s_{g}, \pi\right) \text { if } s_{g}^{I}(\pi)<s_{g}<s_{g}^{D}(\pi) \quad \text { (i.e., in scanning flow). }
$$

Movement along the interior of a scanning curve is reversible, because the configuration of the phases within the pores remains fixed, so that $\partial \pi / \partial t=0$.

The configuration of the phases within the pores changes, however, when the flow state reaches the intersection of the scanning curve with the imbibition curve (the rightmost curve in Fig. 3.1(b)). This happens when $s_{g}$ reaches the value $s_{g}^{I}\left(\pi_{0}\right)$. If $s_{g}$ continues to decrease (so that $1-s_{g}$ increases), then $k_{r g}$ is specified by the function $k_{r g}^{I}$ of $s_{g}$; moreover, $\pi$ changes so that the relationship $s_{g}=s_{g}^{I}(\pi)$ continues to hold. Otherwise, if $s_{g}$ increases, $k_{r g}$ is again specified by $k_{r g}^{S}$ and $\pi$ stays fixed at $\pi_{0}$. Thus

$$
k_{r g}=k_{r g}^{I}\left(s_{g}\right) \text { if } s_{g}=s_{g}^{I}(\pi) \text { and } \partial s_{g} / \partial t<0 \quad \text { (i.e., in imbibition flow). }
$$

Similarly, the pore-level fluid configuration changes when the flow state reaches the intersection of the scanning curve with the drainage curve (the leftmost curve in Fig. 3.1(b)), which occurs when $s_{g}$ reaches the value $s_{g}^{D}\left(\pi_{0}\right)$. If $s_{g}$ continues to increase, then $k_{r g}$ is specified by the function $k_{r g}^{D}$ of $s_{g}$ and $\pi$ changes so that the relationship $s_{g}=s_{g}^{D}(\pi)$ continues to hold. Otherwise, $k_{r g}$ is again specified by $k_{r g}^{S}$ and $\pi$ stays fixed at $\pi_{0}$. Thus

$$
k_{r g}=k_{r g}^{D}\left(s_{g}\right) \text { if } s_{g}=s_{g}^{D}(\pi) \text { and } \partial s_{g} / \partial t>0 \quad \text { (i.e., in drainage flow). }
$$


The qualitative features of the nonwetting phase relative permeability that we assume are the following. (1) The imbibition and drainage curves are placed so that $k_{r g}^{D}\left(s_{g}\right)<k_{r g}^{I}\left(s_{g}\right)$, as is true for many reservoirs. (The physical mechanism underlying this property is the change in the angle made by the gas/water interface at pore walls when the flow reverses.) (2) The scanning curves fill the scanning region without overlapping. (3) The scanning curves intersect the imbibition and drainage curves transversally, with the slopes related by $\partial k_{g}^{s} / \partial s_{g}<d k_{g}^{I} / d s_{g}$ at the imbibition curve and $\partial k_{g}^{s} / \partial s_{g}<d k_{g}^{D} / d s_{g}$ at the drainage curve.

\section{Perturbation-Dependent Riemann Solution for Two-Phase Flow}

This section is devoted to proving the a theorem concerning two-phase flow with hysteresis, which is governed by Eqs. (2.1) in the absence of one of the wetting phases. Let $s$ denote the saturation of the wetting phase that is present, and $f$ its fractional flow, shown in Fig. 4.1(a).

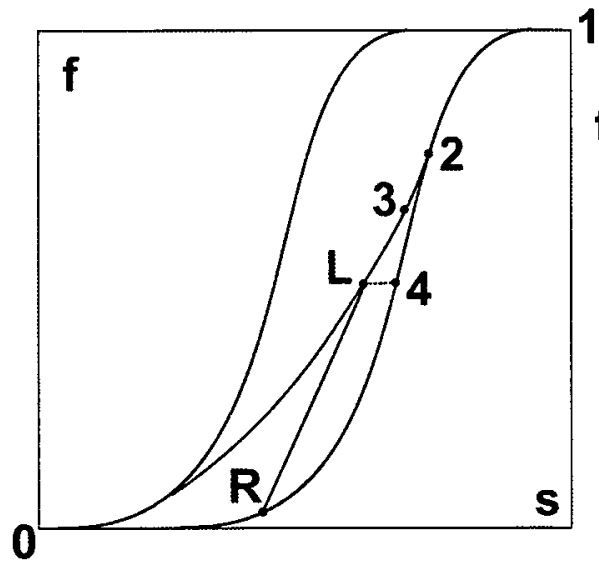

(a)

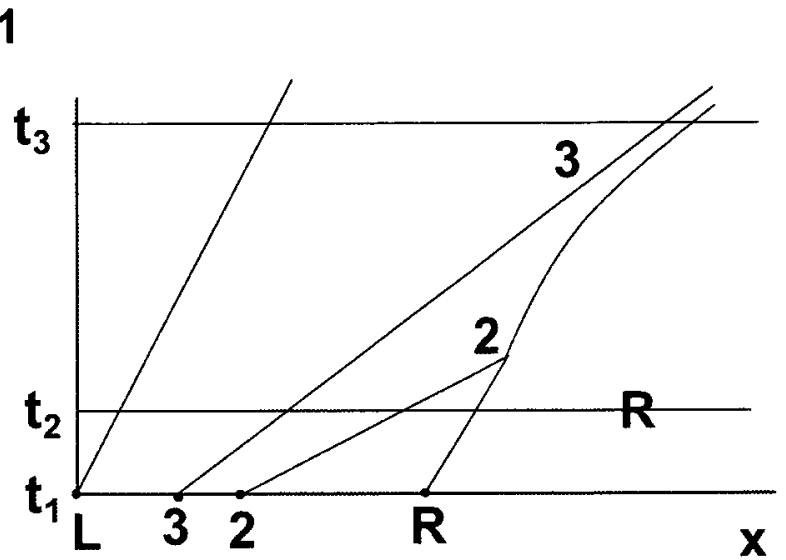

(b)

Figure 4.1. A smoothed Riemann problem. (a) The phase plane. (b) The hodograph plane.

If a Riemann problem $\mathrm{L}->\mathrm{R}$ has data for which $f$ varies monotonically, then its solution, consisting of admissible shock waves and rarefaction fans, is unique [3,5,1]. Moreover, this solution is stable with respect to small monotonic smoothings of the initial discontinuity L->R.

Theorem. The solution of a smoothed Riemann problem $\mathrm{L}->\mathrm{R}$, with data for which is $f$ nonmonotonic and $\mathrm{R}$ belongs to the imbibition curve, consists of a stationary shock from $\mathrm{L}$ to the scanning curve that corresponds to the maximum of $f$ at $t=0$ together with a sequence of shock waves and rarefaction fans along the scanning and imbibition curve.

In the absence of capillary pressure differences, the governing equations are $\partial s / \partial t+\partial f(s, \pi) / \partial x=0$ and $\partial \pi / \partial t=0$. Multiplying by $\partial f / \partial s$ shows that $\partial f / \partial t+c \partial f / \partial x=0$, where $c=\partial f / \partial s$. Thus $f(s, \pi)$ is constant along each characteristic curve $d x / d t=\partial f / \partial s$. The conservation laws allow for shocks with zero velocity leading from one scanning curve to another so long as $f$ does not jump, as well as for shocks along the imbibition and drainage curves that satisfy the Rankine-Hugoniot and Oleinik condition [3, 5, 1]. These shocks are physically admissible (according to analysis [12] of the model discussed in Section 5) and are stable with respect to small perturbation [1].

Figure 4.1 shows the solution of the smoothed Riemann problem L->R with non-monotonic smoothing L-2-R, where L-2 is a segment of the scanning curve and $2-\mathrm{R}$ is a segment of the imbibition curve. The hodograph transformation maps the hodograph plane of Fig. 4.1(b) to the 
phase plane Fig. 4.1(a). The interval [L,2] of the initial data generates the scanning simple wave. The shock $2->\mathrm{R}$ appears after the intersection of characteristic lines that start in the interval [2,R]. This shock enters into the simple wave, and its trajectory tends asymptotically to the characteristic line 3 , where the straight line R-3 is tangent to the scanning curve. Thus the perturbed solution tends to the self-similar path $1-3->R$.

The non-monotonic solution $1-3->\mathrm{R}$ is significantly different from the monotonic solution labeled $1->4->R$ in Fig. 4.1(a). If the point $L$ is located on the drainage curve, the smoothing path follows the drainage curve up to the point 1 and then the imbibition curve up to the point $R$. The solution of the corresponding Riemann problem coincides with the solution that has been obtained in Refs. $[9,10]$ without consideration of scanning hysteresis.

In the case where the point $\mathrm{R}$ belongs to the drainage curve, the solution depends on the minimum value of $f$ in the initial data. Therefore the solution of the Riemann problem depends on the small perturbation of the initial data. The Scanning Hysteresis Model remembers this small perturbation.

\section{The Scanning Hysteresis Model with Relaxation}

As in Ref. [12], we extend the Scanning Hysteresis Model by adding one-sided relaxation. To motivate this extension, consider a flow in which the gas saturation $s_{g}$ is decreased to the critical imbibition saturation $s_{g}^{I}(\pi)$ and continues decreasing. In the SHM, $\pi$ adjusts continuously during imbibition so as to maintain $s_{g}=s_{g}^{I}(\pi)$, meaning that the configuration of the water and oil in the pores is in equilibrium. In reality, the fluid configuration is perturbed from equilibrium by the decrease in $s_{g}$ and is driven back to equilibrium in a short (but nonzero) time interval. Similar behavior occurs during drainage. Accordingly, in the Scanning Hysteresis Model with Relaxation (SHMR), we allow the fluid state to be out of equilibrium, i.e., $s_{g}<s_{g}^{I}(\pi)$ or $s_{g}>s_{g}^{D}(\pi)$, and give dynamics to $\pi$ so that it relaxes to equilibrium on a short time scale $\tau$.

To construct the SHMR, we first extend each scanning curve i.e., $k_{r g}=k_{r g}^{S}\left(s_{g}, \pi\right)$ for fixed $\left.\pi\right)$ beyond the scanning interval $s_{g}^{I}(\pi) \leq s_{g} \leq s_{g}^{D}(\pi)$ on which it is measured. We can choose $k_{r g}^{S}$ to be defined and smooth for all values of $s_{g}$ and $\pi$. This extended function is not unique, but analysis of the model shows that solutions of the SHMR are essentially unaffected by the choice of extension [12], in that the solution is independent of the extension in the limit as $\tau \rightarrow 0^{+}$.

Secondly, we introduce a forcing term $g\left(s_{g}, \pi\right)$ in the differential equation for $\pi$. In the scanning region, $g\left(s_{g}, \pi\right)=0$, so that $\pi$ remains unchanged, just as in the SHM. On the other hand, outside the scanning region, $g\left(s_{g}, \pi\right)$ drives $\pi$ towards the imbibition or the drainage curves, and this tendency grows with increasing deviation of $\pi$ from equilibrium. Solutions of the SHMR are rather insensitive to the choice of the forcing function, so we take $g\left(s_{g}, \pi\right)$ to have a particularly simple form:

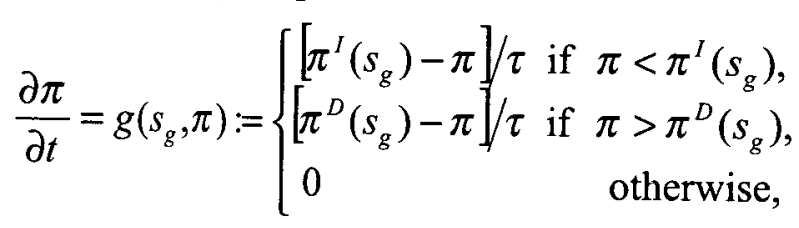


where the constant $\tau>0$ is the relaxation time. Here the function $\pi^{I}$ of $s_{g}$ is defined by solving the equation $s_{g}=s_{g}^{I}(\pi)$ for $\pi$, and $\pi^{D}$ is defined analogously.

The only role of the imbibition and drainage curves in the SHMR is to serve as attractors for external states; no flow orientation is associated a priori with these curves. Nonetheless, this model predicts the correct irreversible behavior during imbibition and drainage [12].

\section{Numerical Experiments}

In this section we present the results of some numerical experiments for one-dimensional threephase flow, both with and without permeability hysteresis. The example flow problems involve injecting water and gas either simultaneously or alternately into a rock core.

\subsection{Model parameters}

We make the following choices to define the specific model that is simulated: (a) $\mu_{w}=0.5$, $\mu_{g}=0.3$, and $\mu_{o}=1.0$; (b) $k_{r w}=s_{w}^{2}$ and $k_{r o}=s_{o}^{2}$; (c) for the model without hysteresis, $k_{r g}=s_{g}^{2}$, whereas for the model with hysteresis, $k_{r g}^{I}=s_{g}^{2}, k_{r g}^{D}=s_{g}^{3}$,

$$
k_{r g}^{S}=\frac{(1-\pi)^{2}}{1-\alpha \pi}\left[1-\alpha\left(1-s_{g}\right)\right] \quad \text { with } \alpha=0.5 \text {, }
$$

and $\tau=0.0003$; and (c) $p_{w o}=\varepsilon\left(2-s_{w}\right)\left(1-s_{w}\right)$ and $p_{g o}=\varepsilon\left(2-s_{g}\right)\left(1-s_{g}\right)$, where $\varepsilon=0.004$ determines the scale for the capillary pressure relative to the pressure drop across the core. For more details, consult Refs. [11,12].

\subsection{Flow problem specification}

For our numerical experiments, the rock core is initially saturated with $10 \%$ water, $75 \%$ oil, and $15 \%$ gas. (This fluid mixture is denoted $S^{R}$.) In the water-alternating-gas (WAG) experiments, the fluid injection at the left side of the core is periodic with period 0.05 , with pure water being injected for a time interval 0.04 and pure gas being injected for the remaining time interval 0.01 . The fractional flows, not the saturations, that must be specified at the boundary. Thus $f_{w}=1$, $f_{o}=0$, and $f_{g}=0$ during $80 \%$ of the period and $f_{w}=0, f_{o}=0$, and $f_{g}=1$ otherwise.

A WAG process is closely related to a process in which water and gas are injected simultaneously, namely the one with the same time-averaged fractional flows. Therefore we also simulated the problem with the fractional flows held fixed at $f_{w}=0.80, f_{o}=0$, and $f_{g}=0.20$.

(This set of fluxes is denoted $F^{L}$.) A WAG process and this associated simultaneous injection problem are expected to have similar solutions, at least after sufficient time has elapsed.

\subsection{Results}

The four flow problems were solved numerically using the linearized Crank-Nicolson method with a grid of 2000 cells. This grid was sufficiently fine to resolve the internal structure of shock waves, which result from the diffusion terms and (when hysteresis is present) the stiff source term. The results at time $t=0.5$ are shown in the figures that follow. Figures 6.1(a)-(d) show plots of $s_{o}$ and $s_{w}+s_{o}=1-s_{g} v s$. position $x$, whereas Figs. 6.2(a)-(d) show the solution plotted in the saturation triangle. 
Figures 6.1(a) and 6.2(a) correspond to the simplest situation, the simultaneous injection problem without hystersis. Interestingly, this solution has three waves, more than the usual two waves. The fastest wave is a Buckley-Leverett shock wave to $S^{R}$ from $S^{B}$. The middle wave, from $S^{A}$ to $S^{B}$, is a transitional shock wave; we refer to Refs. [8,11] for a discussion of the unusual nature of transitional shock waves. The slowest wave, leading from $S^{A}$ to $S^{L}$, is a rarefaction fan of the slower characteristic family. (The saturation state $S^{L}$ is the one for which the flux is $F^{L}$.)

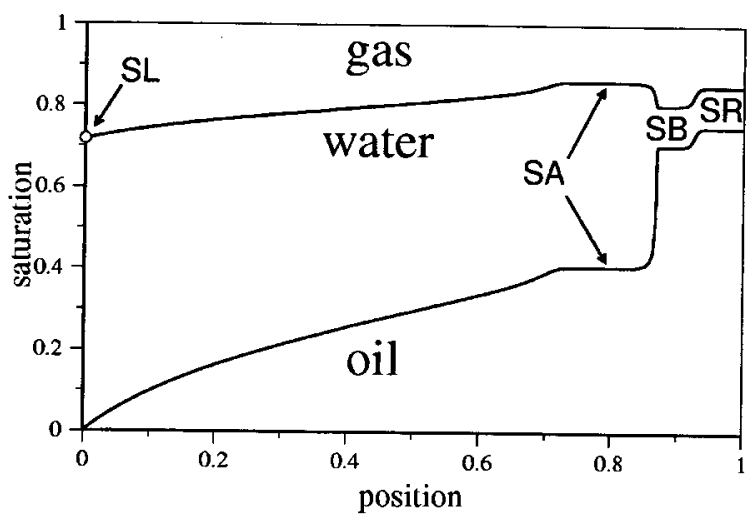

(a)

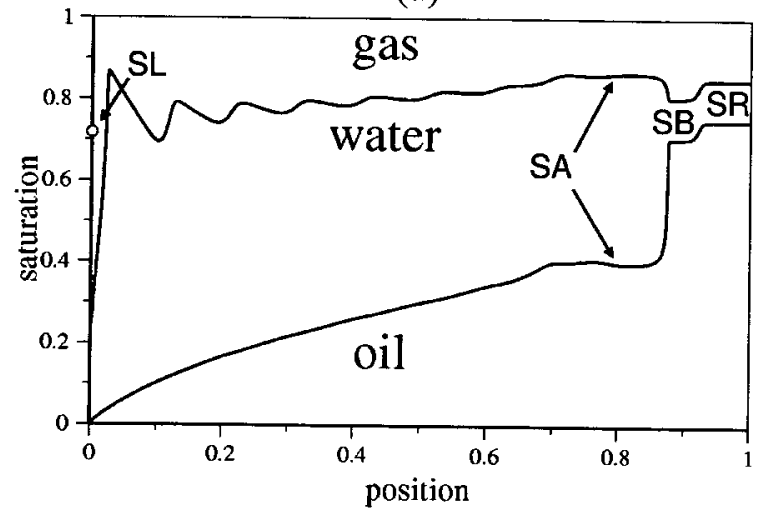

(c)

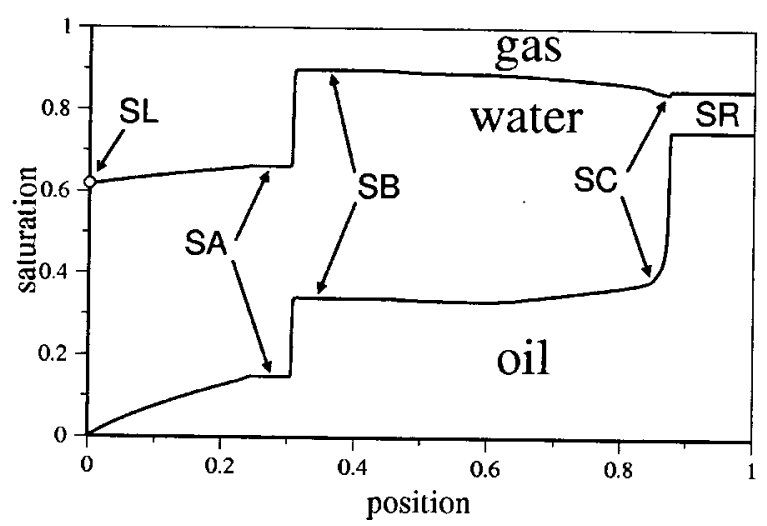

(b)

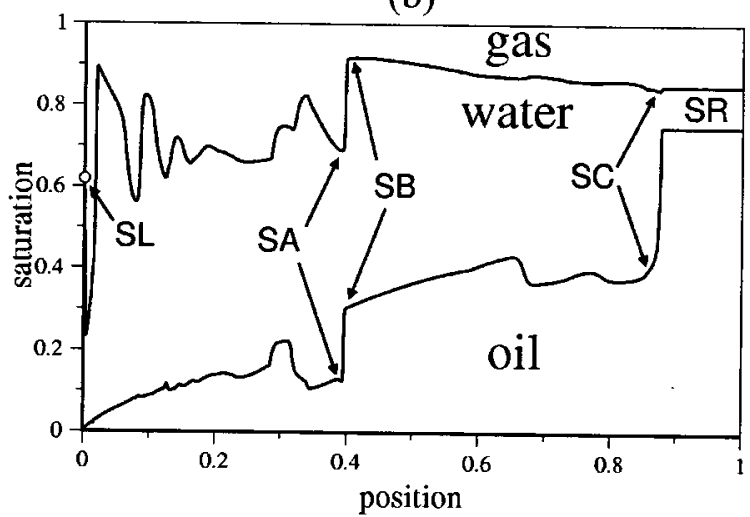

(d)

Figure 6.1. Plots of saturation vs. position at a late time. Simultaneous injection problem (a) without and (b) with hysteresis. WAG problem (c) without and (d) with hysteresis.

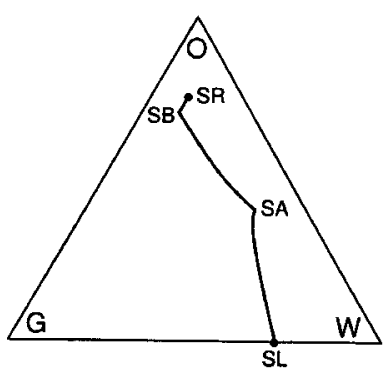

(a)

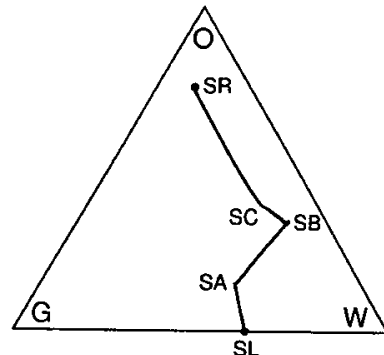

(b)

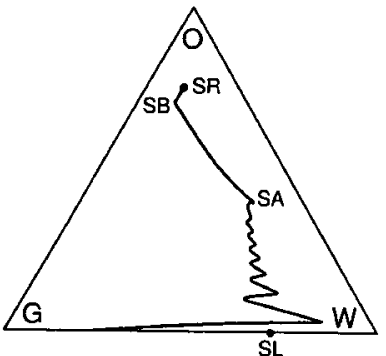

(c)

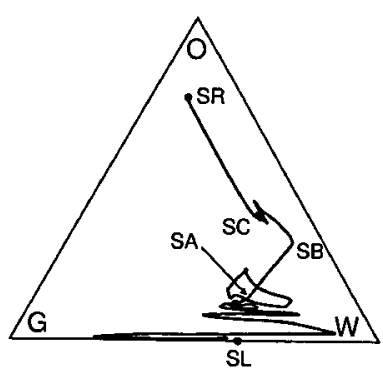

(d)

Figure 6.2. Saturation triangle plots for the solutions in Fig. 6.1. Simultaneous injection problem (a) without and (b) with hysteresis. WAG problem (c) without hysteresis and (d) with hysteresis.

Contrast this solution with that shown in Figs. 6.1(c) and 6.2(c), which correspond to WAG process without hysteresis. The solution is nearly identical, except that the slowest wave is the superposition of two waves, namely a slow rarefaction fan from $S^{A}$ to $S^{L}$ and an oscillating 
wave in the fast characteristic mode. The latter wave, which is generated by the injected saturation oscillations, decays as it moves into the core. Because of its large amplitude, this oscillating wave is affected by the nonlinearity of the equations. In fact, as this wave propagates into the core, some parts sharpen under convergence of characteristics, thereby forming shock waves, whereas other parts spread under divergence of characteristics, forming rarefaction fans. Furthermore, the resulting shock waves and rarefaction fans interact, weakening each other as they move away from the injection point.

In summary, the oil saturation profile for the WAG solution is practically indistinguishable from that for the simultaneous injection solution for the time-averaged flux $F^{L}$ and the initial state $S^{R}$, and the water saturation profile is a superposition of a nonlinear, decaying oscillatory wave on the corresponding profile from the simultaneous injection solution.

The effect of hysteresis on the simultaneous injection injection process is illustrated in Figs. 6.1(b) and 6.2(b). There are three wave groups in the solution. The fast wave group consists of a Buckley-Leverett shock wave from $S^{R}$ to $S^{C}$ preceded by an adjoining fast-family rarefaction wave from $S^{C}$ to $S^{B}$. The slowest wave group is a rarefaction fan from $S^{A}$ to $S^{L}$. In the middle is a new type of shock wave from $S^{B}$ to $S^{A}$. To identify this wave, consider the plot of $k_{g}$ vs. $s_{g}$ shown in Fig. 6.3. This plot demonstrates that $S^{B}$ lies on the imbibition curve and $S^{A}$ lies on the drainage curve, so that this shock wave is a imbibition-to-drainage shock wave. It also shows the internal structure of this shock wave, which closely follows a scanning curve, then extends outside the scanning region, and finally returns to the drainage curve, which is as expected, according to the analysis of Ref. [12].

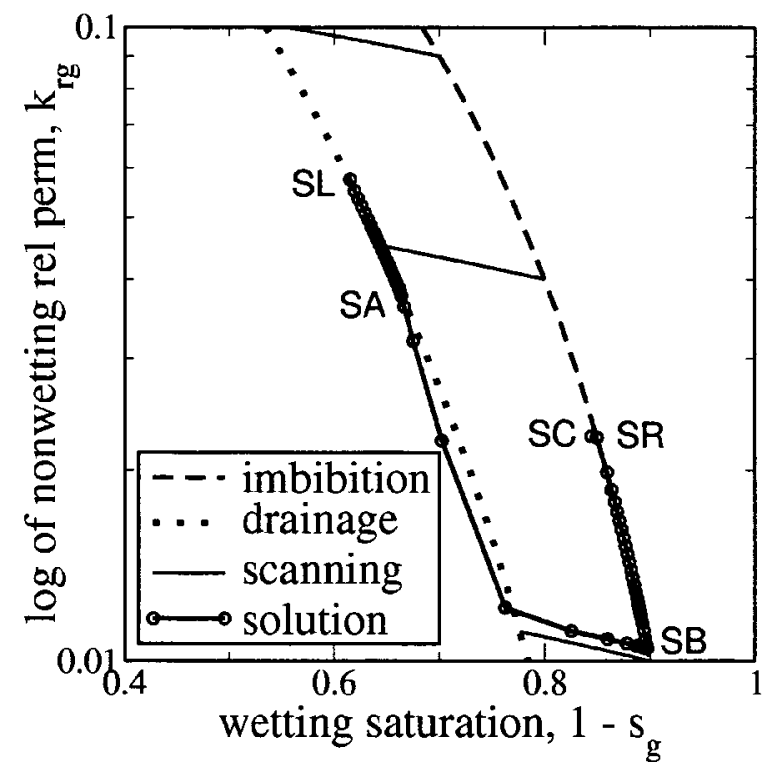

Figure 6.3. The simultaneous injection solution with hysteresis (Figs. 6.1(b) and 6.2(b)).

Finally, consider the WAG injection process with hysteresis, which is shown in Figs. 6.1(d) and 6.2(d). Although the structure of the solution is complicated, it is easily understood using the insights gained from the simpler flow problems. Indeed, this solution resembles the simultaneous injection solution with hysteresis together with superimposed oscillating waves. The imbibitionto-drainage shock, however, has a somewhat different location as a result of its interaction with oscillatory waves; this difference in location is expected to decrease at later time. 


\section{Summary}

In this paper, we modeled permeability hysteresis using the empirical scanning curves to define the fractional flow functions, a single new variable to record the flow history, and the empirical imbibition and drainage curves define the relaxation dynamics of this variable. We also numerically simulated some examples of one-dimensional three-phase flow to illustrate the effects of hysteresis on simultaneous and alternating injection of water and gas into a rock core. The presence of the drainage-to-imbibition shock wave is the primary new feature caused by hysteresis. This wave is a sharp oil-bank moving at a significantly lower speed than the primary Buckley-Leverett oil-bank. Because it brings the oil saturation down to a low value, this wave reduces the amount of oil left in the reservoir after it arrives at the production point. Thus a WAG strategy can be more efficient when the flow exhibits hysteresis.

Acknowledgements

This work was supported in part by: CNPq under Grant 300204/83-3; CNPq under Grant 523258/95-0; FINEP under Grant 77.97.0315.00; NSF under Grant DMS-9732876; DOE under Grant DE-FG02-90ER25084; and ARO under Grant 38338-MA.

\section{References}

[1] P. Bedrikovetsky, Mathematical Theory of Oil \& Gas Recovery, Kluwer Academic Publishers, London, 1993.

[2] P. Bedrikovetsky, D. Marchesin, and P. R.Ballin, Mathematical theory for two-phase displacement with hysteresis (with applications to WAG injection), $\mathrm{V}$ European Conference on Mathematics in Oil Recovery, Austria, Leoben, Sept. 3-6, 1996.

[3] P. Bedrikovetsky, D. Marchesin, and P. R. Ballin, Mathematical model for immiscible displacement honouring hysteresis, SPE 30132, Fourth Latin American and Caribbean Petroleum Engineering Conference, Port of Spain, Trinidad-Tobago, pp. 557-575, 1996.

[4] E. Braun and R. Holland, Relative permeability hysteresis: Laboratory measurements and a conceptual model, SPE Reservoir Engineering, August, pp. 222-228, 1997.

[5] K. Furati, The solution of the Riemann problem for a hyperbolic system modeling polymer flooding with hysteresis, J. Math. Anal. Appl., vol. 206, pp. 205-233, 1997.

[6] K. Furati, A hysteretic polymer flooding model, Ph.D. Thesis, Duke University, Department of Mathematics, 1995.

[7] K.Furati, Effects of relative permeability hysteresis dependence on two-phase flow in porous media, Transport in Porous Media, vol. 28, pp. 181-203, 1997.

[8] E. Isaacson, D. Marchesin, and B. Plohr, Transitional waves for conservation laws, SIAM J. Math. Anal., vol. 21, 837-866, 1990.

[9] D. Marchesin, H. B. Medeiros, and P. J. Paes-Leme, A model for two-phase flow with hysteresis, Contemporary Mathematics, vol. 60, pp. 89-107, 1987.

[10] D. Marchesin, H. B. Medeiros, P. J. Paes-Leme, Hysteresis in two-phase flow: A simple mathematical model, Comput. Appl. Math., vol. 17, pp. 81-99, 1998.

[11] D. Marchesin and B. Plohr, Wave structure in WAG recovery, SPE J., vol. 6, pp. 209-219, 2001.

[12] B. Plohr, D. Marchesin, P. Bedrikovetsky, and P. Krause, Modeling hysteresis in porous media flow via relaxation, Comput. Geosciences, vol. 5, pp. 225-256, 2001.

$8^{\text {th }}$ European Conference on the Mathematics of Oil Recovery - Freiberg, Germany, 3 - 6 September 2002 
